\begin{abstract}
Background: Assessment options are extremely limited for the evaluation of treatment outcome in a specific phobia of vomiting. We aimed to assist researchers and clinicians in measurement of cognitive processes and behaviors that are characteristic of the disorder for treatment planning and outcome measurement. Method: We developed the Specific Phobia of Vomiting Inventory (SPOVI). A series of measures, including the SPOVI, were given to two groups: a group diagnosed with a specific phobia of vomiting and a community control group. Item characteristics, reliability, and factor structure were analysed. Convergent validity with measures of related constructs was determined. Results: The SPOVI was found to have good reliability and validity in the measurement of a phobia of vomiting. The scale has a two-factor structure, with one factor characterised by avoidance symptoms and a second factor comprised of threat monitoring. It is sensitive to change during treatment.

Conclusions: The results provide initial evidence of the psychometric qualities of the SPOVI and its suitability for use in clinical practice and research.
\end{abstract}

Keywords: specific phobia; vomiting; inventory; questionnaire, emetophobia 


\section{Development of an inventory to measure specific phobia of vomiting (emetophobia).}

A Specific Phobia of Vomiting or emetophobia is a clinical condition characterised by a preoccupation with and fear of vomiting. Emetophobia is a neglected area of research. The condition appears uncommon, with a prevalence of $0.1 \%$ in the only epidemiological survey that has specifically asked about a phobia of vomiting (Becker et al., 2007). Previous estimates of prevalence may, however, be underestimates as the symptoms may be confused with symptoms of health anxiety, obsessive-compulsive disorder, social phobia, panic disorder, and anorexia nervosa (Boschen, 2007; Veale, 2009). Thus some people with SPOV may have checking compulsions (for example excessive checking that food is not out of date or that others are not ill in order to reduce the risk of vomiting). However an additional diagnosis of co-morbid obsessive-compulsive disorder would only be used when the obsessions are not restricted to fears of vomiting. This is the same for health anxiety in which people with SPOV may worry and seek reassurance about themselves or others not vomiting. However a diagnosis of health anxiety is only made if the fears of being ill are not confined to vomiting. Symptoms of nausea and misinterpretation as evidence of vomiting might arise in, for example, panic disorder and agoraphobia, but these are associated with other symptoms of panic (e.g. palpitations for heart attack, shortness of breath for stopping breathing). Similar issues exist for specific phobias linked to other bodily functions such as choking or incontinence. At the symptom level, anxiety about vomiting has been reported in as many as $3.1 \%$ of males and 7\% of females (Kirkpatrick \& Berg, 1981, cited in Philips, 1985).

Despite being uncommon compared to specific phobias in general, emetophobia can lead to significant impairment, and reduced quality of life. Female- 
specific impairments include avoiding a desired pregnancy out of fear of morning sickness and the propensity of babies to vomit also causes problems (Veale \& Lambrou, 2006). Individuals with emetophobia usually report early onset of the condition, with a chronic course lasting over many years, and very few periods of remission (Lipsitz, Fyer, Paterniti, \& Klein, 2001). Individuals with emetophobia also report significant distress due to their symptoms (Lipsitz et al., 2011). Fear and avoidance associated with emetophobia impairs functioning in a range of ways, such as being significantly underweight from dietary restriction and misdiagnosis of anorexia nervosa (Veale, Costa, Murphy, \& Ellison, 2012), avoiding social or public situations where there may be a risk of vomiting. There are frequent safety-seeking behaviors which may be either overt or a covert mental act with the aim of preventing oneself or others being sick. Overt behaviors include checking of 'sell by' dates and freshness of food, reassurance seeking, excessive cooking of food, excessive washing of hands or cleaning with anti-bacterial sprays, or drinking bottled water to check no food is coming up. Covert acts include a person mentally reviewing her or others' actions and reassuring herself that she will not vomit.

There is some evidence for associative learning in emetophobia with aversive consequences of vomiting or an unrelated life event (Veale, Murphy, Ellison, Kanakam, \& Costa (2012). It suggests a model of autobiographical memories of vomiting that have lost a time perspective and context, which are being reactivated with cues for vomiting. There is a gender bias towards woman in emetophobia. This does not necessarily indicate for a higher genetic contribution to a disorder unless a rare mechanism like an X-chromosome linked disorder or a mitochondrial DNA transmission is hypothesized. The higher prevalence in women may be a reflection of 
an increased disgust sensitivity and preparedness as a result of a greater responsibility for the care of children.

Treatment outcome studies for emetophobia are limited to clinical case reports although earlier studies often do not describe the diagnostic criteria that were used or give much detail on the outcome. Procedures used include video-taped exposure to others vomiting (Philips, 1985); graded exposure to simulated vomiting (McFadyen \& Wyness, 1983); exposure (“flooding”) to vomiting under hypnosis (Wijesinghe, 1974); exposure to nausea (Lesage \& Lamontagne, 2003); exposure to interceptive cues of vomiting (Hunter \& Antony, 2009); systemic behavior therapy (O'Connor, 1983); competence imagery (Moran \& O’Brien, 2005); psychodynamic therapy and exposure (Ritow, 1979); hypnotherapy (McKenzie, 1994); psychotherapy (Manassis \& Kalman, 1990). None of these treatment outcome studies of emetophobia have used a standardized, psychometrically validated measure of emetophobic related symptoms or of a specific phobia (Antony, 2001). They have utilized a diverse array of outcome measures such as anxiety ratings to vomiting cues or simulated vomiting (McFadyen \& Wyness, 1983; Philips 1985), diary records of episodes of nausea (Lesage \& Lemontagne, 1985) or non-specific measures of anxiety (Moran \& O’Brien, 2005; Hunter \& Antony, 2009). Several have not used any outcome measures at all (Wijesinghe, 1974; O’Connor, 1983; Manassis \& Kalman, 1990; McKenzie, 1994; Ritow, 1979). The lack of any a specific measure makes comparison between outcomes of different therapies very difficult (Jacobsen, Roberts, Berns, \& McGlinchey, 1999). The use of different measures also prevents aggregation of results from the small number of existing studies for use in statistical procedures such as meta-analysis. More general anxiety measures or inventories may allow for the 
calculation of the effect size of treatments on these general symptoms, but do not allow for evaluation of the effect of treatment on emetophobia symptoms.

The present study addresses the lack of established measures of emetophobia by validating a self-report inventory, the Specific Phobia of Vomiting Inventory (SPOVI). The SPOVI is a self-report measure that focuses on the cognitive processes and avoidance behaviors that are characteristic of the disorder. We desired a scale that was free, brief and suitable for the assessment of symptom change during treatment. Furthermore, we sought to create a scale that would assist clinicians in identifying the most frequent cognitive processes and behaviors that theoretically maintain the symptoms, and which therefore could be targeted in therapy.

\section{Method}

\section{Participants}

Participants of both sexes were recruited for the clinical sample; for the control group, recruitment was matched for gender to balance the significant overrepresentation of females in the clinical cohort.

Emetophobia group. The emetophobia group consisted of 95 participants with DSM-IV diagnosis of emetophobia ( 89 female, 6 male), 25 of whom were recruited from a clinical setting, and a further 70 from the Internet. There were 90 non-emetophobic participants from the community group ( 87 female, 3 male).

Of those participants in the emetophobia cohort, 60 (63.2\%) reported no comorbid diagnoses, $20(21.1 \%)$ had one comorbid diagnosis and $15(15.8 \%)$ had two 
or more comorbid diagnoses. The most common comorbidities among the participants were depression $(n=8,8.4 \%)$, GAD $(n=8,8.4 \%)$, OCD $(n=6,6.3 \%)$, somatisation $(n=5,5.3 \%)$, panic disorder $(n=4,4.2 \%)$, social phobia $(n=4,4.2 \%)$, agoraphobia $(n=2,2.2 \%)$, health anxiety $(n=1,1.1 \%)$, and other specific phobia $(n=1,1.1 \%)$.

Community group. A community sample was obtained for the purpose of comparison with our clinical cohort on the new measure. A community group $(N=$ 90) was identified on the Mind Search database at the Institute of Psychiatry, Kings College London. This database contains details for over 3,500 individuals in the local community who have volunteered to participate in psychological or psychiatric research.

Participants were excluded from both groups if they were at greater risk of vomiting. This was to ensure that the control group were likely to have a similar frequency of vomiting as the emetophobia group. The exclusion criteria were: an eating disorder with self-induced vomiting; suicidal intent and a history of taking an overdose that could induce vomiting; regular binge drinking and vomiting; use of illegal substances that might cause vomiting (e.g., opiates); current use of medication or other treatments that can cause vomiting (e.g., chemotherapy; radiotherapy); current medical problems that could cause vomiting (e.g., peptic ulcer, cancer, migraine); or current pregnancy.

There were no significant differences in age $(t=0.08, d f=182, p=.94)$ or gender distribution $\left(\chi^{2}=2.53, d f=1, p=.11\right)$ between the two groups. Demographic details of both samples are presented in Table 1.

\section{Procedure}


The diagnosis of a specific phobia of vomiting was made using the Structured Clinical Interview for DSM Disorders (SCID) for DSM-IV (APA, 1994). The Psychiatric Diagnostic Screening Questionnaire (PDSQ; Zimmerman \& Mattia, 2001) was used to identify possible alternative diagnoses and co-morbid conditions before using the SCID. A clinical research worker trained in the use of the SCID interviewed participants recruited via the Internet over the telephone. The diagnosis of a specific phobia of vomiting in patients recruited within the clinical setting was determined in the context of a clinical assessment by a psychologist or psychiatrist using the SCID. After they consented, they completed all the questionnaires.

All the participants with emetophobia recruited from the Internet were invited to be retested but only 31 agreed and provided retest data. Those retested were not significantly different in emetophobia severity to those who were not retested $(t=$ $0.31, p=.76)$. The retesting was administered online. The test-retest reliability was not conducted in the community group.

Eight participants from the emetophobia group were followed up during treatment. They were chosen because they had funding and were able to have treatment in our setting. Therapists followed a treatment protocol described by Veale (2009) that included a formulation and procedures that included (a) imagery rescripting to past experiences of vomiting, (b) exposure to situations and activities associated with vomiting (Wolitzky-Taylor, Horowitz, Powers, \& Telch, 2008), (c) modifying safety seeking behaviors and cognitive processes such as reducing selffocussed attention, checking and worrying. They attended weekly for up to 12 one hour sessions. These participants completed the SPOVI, PHQ9, and GAD7 at pretreatment, mid-treatment and at the end of therapy. 
All participants received a gift voucher of $£ 10$ after completion of the questionnaire. SelectSurveyASP (TM) version 8.1.1 was used to create a web-based version of the questionnaires completed for the control group and participants in the emetophobia group who were recruited over the Internet. The format and structure of the questions were identical to the paper version used in the clinical setting.

\section{Materials}

The following questionnaires were completed by each participant in the emetophobia and community control group, in addition to the gathering of basic demographic data.

Specific Phobia of Vomiting Inventory (SPOVI). The SPOVI consists of 14 items each scored on a Likert-type scale for frequency from 0 (not at all) to 4 (all the time). The items refer to the past week. The total score ranges from 0 to 56 with a higher score reflecting greater frequency of emetophobia-related symptoms.

Items for the SPOVI were generated from interviews in a previous study on the psychopathology of a specific phobia of vomiting in which we identified the characteristic cognitive processes and behaviors (Veale \& Lambrou, 2006; Price, Veale, \& Brewin, 2012). Items followed a theoretical model for the maintenance of symptoms of a specific phobia of vomiting (Veale, 2009). We also drew upon established trans-diagnostic processes that occur in anxiety disorders (Harvey, Watkins, Mansell, \& Shoran, 2004). A process of iteration occurred so that experienced clinicians and patients with emetophobia reviewed the wording of the draft version. Where the meaning or context of an item was unclear, it was accordingly modified. It was pilot tested in people with specific phobia of vomiting before the final version was used for the study. 
Disgust Scale Revised (DS-R; Olatunji et al., 2007). The DS-R is a selfreport questionnaire originally developed by Haidt, McCauley, and Rozin (1994) as a general tool for the study of disgust. It has been previously used to measure individual differences in sensitivity to disgust, and to examine the relations among different kinds of disgust. Van Overveld et al. (2008) have previously reported that people with emetophobia have increased levels of disgust propensity and sensitivity. The DS-R is a shortened version of the original Disgust Scale, with the number of items reduced from 32 to 25, and the number of subscales reduced from eight to three (core disgust, animal-reminder disgust, and contamination disgust). The response format for items is a 5-point Likert scale from 0 (not disgusting at all/strongly disagree) to 4 (extremely disgusting/strongly agree). The total score is in the possible range of 0 to 100 . Cronbach's alpha in the current sample was .87.

\section{Obsessive Compulsive Inventory (OCI; Foa, Kozak, Salkovskis, Coles, \&}

Amir, 1998). The OCI is a 42-item self-report measure of obsessive-compulsive disorder symptoms. In the current study, participants rated each item for distress on a 5-point Likert scale from 0 (not at all) to 4 (extremely), yielding a possible range of 0 to 168 . Distress can also be rated separately for each of seven subscales: Washing, Checking, Doubting, Ordering, Obsessing, Hoarding, and Neutralizing but these were not utilized because of the risk of generating false positive associations from more statistical analyses. The Cronbach's alpha of the OCI in the current sample was .96.

\section{Health Anxiety Inventory (HAI; Salkovskis, Rimes, Warwick, \& Clark,}

2002). The HAI is a self-rated measure of health anxiety that is sensitive across the full range of intensity (from mild concern to frank hypochondriasis). The HAI differentiates people suffering from health anxiety from those who have actual physical illness, but who are not excessively concerned about their health. It also 
encompasses the full range of clinical symptoms characteristic of clinical hypochondriasis. We used the short version (14 items) of the scale. The possible range for the total is 0-42. The Cronbach's alpha in the current sample was .95.

Emetophobia Questionnaire (EmetQ-13) (Reddell, 2006). The EmetQ-13 is a 13-item scale that is answered on a Likert-scale from 1 ('strongly disagree') to 5 ('strongly agree') with a possible range of 13 - 65. The EmetQ-13 has a clear threefactor structure. It has good internal consistency $(\alpha=.82$ in the clinical sample, and $\alpha$ $=.85$ in a control sample), and one-week test-retest reliability $\left(r_{\mathrm{xx}}=.76\right)$. The EmetQ13 also shows excellent ability to differentiate individuals with emetophobia from non-clinical controls. The questionnaire focuses on avoidance of vomit-related situations (e.g., 'I avoid places where others may vomit') and beliefs about nausea (e.g. If I see vomit, I may be sick myself'). The EmetQ-13 and the SPOVI were developed independently in Australia and the United Kingdom, respectively.

PHQ-9 Depression Severity (Kroenke \& Spitzer, 2002). The Patient Health Questionnaire (PHQ) is a self-report version of the PRIME-MD diagnostic instrument for common mental disorders. The PHQ-9 is the depression module, which scores each of the 9 DSM-IV criteria as "0" (not at all) to " 3 " (nearly every day). The PHQ-9 total score for the 9 items ranges from 0 to 27 . The PHQ-9 demonstrated high internal consistency in the current sample (Cronbach's $\alpha=.93$ ).

\section{Generalized Anxiety Disorder assessment (GAD-7; Spitzer, Kroenke,}

Williams, \& Löwe, 2006). The GAD-7 is designed primarily as a screening and severity measure for symptoms of generalized anxiety disorder. The scale is scored from " 0 " (not at all) to " 3 " (nearly every day) and the total score for the 7 items ranges from 0 to 21 . The internal consistency of the GAD-7 on the current study was high (Cronbach's $\alpha=.94)$. 


\section{Work and Social Adjustment Scale (WSAS; Mundt, Marks, Shear, \&}

Greist, 2002). Emetophobia participants completed the WSAS, which was adapted to focus on the fear of vomiting. This scale measures impairment in functioning, and has five items: "To what extent does your fear of vomiting currently have an effect on your (a) ability to work or study; (b) home management; (c) social life; (d) leisure activities; and (e) relationship with a partner. Items are scored between 0 ("Not at all") and 8 ("Extremely"), and the possible range for the total score across was 0 to 40. Cronbach's alpha in our sample was .69.

\section{Results}

Prior to analysis, data was examined to ensure suitability for statistical analysis. All assumptions were satisfied unless otherwise stated. In the event of violation of any statistical assumption, alternative analytic methods (e.g., nonparametric statistics) were used. Mean imputation was used for all questionnaires where only one item was missing. Missing values were not replaced from questionnaires where more than one item was missing.

\section{Tests of Group Equivalence}

Comparisons on a range of clinical variables were conducted between those individuals with a specific phobia of vomiting recruited from the Internet and those who were seen face-to-face in a clinical setting. These two emetophobia subgroups did not differ on total SPOVI score $(t=1.14, d f=93, p=.26)$, total EmetQ-13 score $(t$ $=0.82, d f=89, p=.42)$, total DS-R score $(t=0.35, d f=87, p=.73)$, total OCI score $(t=0.37, d f=80, p=.71)$, PHQ-9 score $(t=0.17, d f=83, p=.86)$, GAD-7 score $(t=$ $1.42, d f=87, p=.16)$, or WSAS score $(t=0.37, d f=83, p=.71)$. The group seen in a clinical setting reported a significantly higher HAI total score $(M=22.76, S D=$ 
8.36) than the group recruited from the Internet $(M=18.64, S D=7.64, t=2.05, d f=$ $74, p=.044)$. As only one scale differed between the two groups, it was decided that the specific phobia of vomiting group could be treated as a single cohort.

Factor structure. There was insufficient variance in the non-emetophobic group to permit meaningful factor analysis, and so factor analysis was conducted with the emetophobic group only. Horn's parallel factor analysis (Horn, 1965) was used to examine the factor structure of the SPOVI. This was computed using FACTOR version 8.02 (Lorenzo-Seva \& Ferrando, 2006). This method was chosen as it is more accurate than other methods in determining the number of components/factors to extract during factor analysis (Wilson \& Cooper, 2008; Zwick \& Velicer, 1986). Factors were extracted using a principal components extraction method, with this being followed by promax rotation, permitting correlation between the emergent factors. Bartlett's statistic $(714.3, d f=91, p<.001)$ and the Kaiser-Meyer-Olkin test $(K M O=.874)$ indicated that the sample was adequate for factor analysis. The factor analysis in the emetophobia group suggested 2 latent factors, which together accounted for $58.67 \%$ of the overall item variance. Table 4 shows the loadings of the items on each of the two emergent factors. All items demonstrated adequate communality, and returned factor loadings of $>.40$ on only one factor (see Table 2 ). The first factor represented avoidance and second factor represented of threat monitoring and control of symptoms. The Avoidance factor comprised a wide range of avoidance behaviors including avoiding or trying to control people, objects, situations, certain food, thoughts and images because of the fear of vomiting. The Threat Monitoring included processes such as worrying about vomiting; mental planning how to stop oneself from vomiting; attempting to find reasons for nausea; and being excessively self-focussed on monitoring the feeling of being ill. 
When subscales were created from the items using the factor structure in Table 2, the internal consistency (Cronbach's $\alpha$ ) in the emetophobic sample was .85 and .88 for the first and second subscales, respectively.

Internal consistency. The Cronbach's alpha of the SPOVI for the emetophobia group was .91. In the community group, Cronbach's alpha was calculated as .81.

Test-retest reliability. Test-retest reliability was calculated by examining the correlation between scores from two administrations of the SPOVI occurring one week apart, in a subsample of 31 individuals from the emotophobia group who were not currently undergoing treatment for their phobia of vomiting. A one-week hiatus period was chosen as this would be consistent with the use of the SPOVI in weekly therapy sessions as a measure of treatment effect. The SPOVI showed good one-week stability $(r=.85, p<.001)$, and showed no significant change between the two administrations $\left(M_{\text {Time1 }}=29.52, S D_{\text {Time1 }}=14.60, M_{\text {Time2 }}=30.39, S D_{\text {Time2 }}=13.57, t=\right.$ $0.62, d f=30, p=.54)$.

Group Differences. SPOVI scores were significantly higher in the emetophobia group $(M=30.62, S D=12.95)$ compared with the community controls $(M=1.53, S D=3.49, t=20.61, d f=183, p<.001)$. Table 3 provides the mean and standard deviation for all the scales in the emetophobia and community group.

We used the total SPOVI score in an analysis of the sensitivity and specificity of the measure in determining diagnostic status, as well as a receiver operating curve (ROC) analysis. Receiver Operating Characteristics (ROC) analysis was used to illustrate graphically the sensitivity and specificity of the SPOVI in discriminating between patients with emetophobia and the control group at different cut-off values. The area under the curve (AUC) for this analysis was .994 $(\mathrm{p}<.001)$ indicating that the 
SPOVI is a very accurate diagnostic test. To determine the optimal cut-off value of the SPOVI for the identification of subjects with emetophobia, sensitivity and specificity was computed for different cut-off scores. A range of cut-off scores is presented in Table 4, along with the sensitivity and specificity in identifying individuals with specific phobia of vomiting (as distinct from the control group). We suggest a cut-off score of $>10$ as the best compromise between sensitivity and specificity in detecting the presence of emetophobia.

Concurrent validity. Across the whole sample, the concurrent validity against EmetQ-13 was high $(r=.82, p<.001)$, indicating that the two measures are assessing similar constructs.

Convergent validity. In the combined sample, the SPOVI was moderately correlated with the Disgust Scale Revised $(r=.36, p<.001)$, indicating that high levels of symptoms of emetophobia were associated with higher disgust sensitivity. The SPOVI correlated moderately with the OCI total score $(r=0.49, p<.001)$ and highly with the Health Anxiety Inventory $(r=.78, p<.001)$, indicating a strong relationship between fears of vomiting and especially health anxiety. There was also a high correlation with the GAD-7 scale $(r=.59, p<.001)$, and moderate correlation with the PHQ-9 $(r=.49, p<.001)$ showing that higher scores on the SPOVI are associated with greater symptoms of generalized anxiety and depression. The SPOVI also correlated moderately with the Work and Social Adjustment Scale $(r=.52, p<$ $.001)$

Sensitivity to treatment effect. The eight participants in the emetophobia group who were treated for their phobia had a mean age of 30.2 and standard deviation of 14.4. SPOVI scores were tracked during treatment in eight participants in order to determine sensitivity to change. 
Figure 2 shows change on the SPOVI for the 8 participants receiving Cognitive Behavior Therapy. Non-parametric comparisons (Wilcoxin signed ranks tests) were used between pre-treatment and post-treatment scores due to the small samples size. Mean SPOVI scores reduced significantly during treatment $(Z=2.52, p$ $=.012$ ). The mean score reduced from 37.4 (standard deviation 14.1) to post treatment score at 14.4 (standard deviation 12.5). The PHQ-9 measure of depression also reduced during treatment $(Z=2.21, p=.027)$, as did the GAD-7 measure of generalized anxiety symptoms $(Z=2.38, p=.018)$.

\section{Discussion}

The current study examined the psychometric properties of a novel self-report measure of the symptoms of emetophobia. Analysis of SPOVI results in clinical and normative samples established the inventory's reliability, validity, and factor structure.

The two assessments of reliability of the SPOVI were measures of internal consistency and test-retest stability. The SPOVI demonstrated good internal consistency, demonstrating that the scale was measuring a coherent single construct. The SPOVI was also shown to have adequate temporal stability as measured over a one week test-retest period.

Evidence for the validity of the SPOVI in measuring symptoms of emetophobia came from correlations with another measure of emetophobia and emetophobic-related constructs, theory-consistent group differences, and sensitivity to the effects of treatment on symptom change. The SPOVI showed significant and moderate-to-strong correlations with measurements of health anxiety and OCD symptoms, as well as measures of GAD and depression symptoms. Higher SPOVI 
scores were also associated with reductions in work and social adjustment. Importantly, the SPOVI correlated strongly with another self-report measure of emetophobic symptoms. Total SPOVI scores were significantly higher in individuals with emetophobia compared to a non-clinical normative sample. Individuals treated for emetophobia showed significant reductions in SPOVI scores over the course of their intervention.

There were two factors identified which we have labelled "avoidance" and "threat monitoring" and may be used as subscales. They are theoretically meaningful and consistent with phenomenology of emetophobia. All specific phobias are characterized by avoidance of cues that may lead to activation of fear and disgust related to the phobia. Threat monitoring and mental planning interacts with avoidance. Thus when avoidance behaviour is marked and the threat reduces then the process of threat monitoring and vigilance decreases. When avoidance behaviour decreases then threat monitoring and vigilance increase.

The only other measurement tool for emetophobia, the EmetQ-13, was developed independently from the SPOVI, and covers a different range of symptoms. The factor structure of the EmetQ-13, however, also shows a separation of avoidance symptoms from other symptoms, although for the EmetQ-13, these avoidance symptoms are separated into two distinct factors: avoidance of situations and movement, and avoidance of people who may vomit. The EmetQ also includes a third factor on misinterpretation of seeing or smelling vomit in anticipation of oneself vomiting. This factor does not occur on the SPOVI. However the advantage of the SPOVI is the measurement of the process of the threat monitoring which does not occur on the EmetQ-13. Both scales are brief and may be used concurrently. 


\section{Implications}

The immediate implication from the current work is that the SPOVI now exists as a potential measure for use in the assessment and quantification of severity of symptoms of emetophobia. Such a measure can be used for clinical purposes such as the assessment of initial severity of symptoms, to measure changes in symptom severity over the course of therapy and to identify specific processes to target in therapy. It may be also of interest to know whether the factor of avoidance or threat monitoring changes first or which of the two factors leads to greater change in treatment. In research settings, the SPOVI may also be used to assess the impact of treatments for SPOVI in case studies, and in larger controlled and uncontrolled trials with larger samples. The SPOVI is brief, and so can be used weekly to assess symptoms and symptom change in both clinical and research settings.

The scale may assist in identifying people in the community with emetophobia to obtain better data on prevalence. The prevalence of emetophobia may be uncommon but researchers and people with emetophobia need a scale to use in future controlled trials and to estimate effect size of different treatments and audit outcome.

More broadly, our results provide interesting information about the association of emetophobia symptoms with the symptoms of other related conditions. Individuals with fears of vomiting were also more likely to report higher levels of disgust sensitivity, confirming the finding of van Overveld and colleagues (2008). Symptoms of emetophobia were most strongly associated with symptoms of health anxiety, which reflect the overlapping concern with developing a physical illness (Salkovskis et al, 2002). As a group the mean score on the HAI crosses the suggested cut off score for hypochondriacal disorder (Salkovskis et al, 2002). This finding was not unexpected as people with emetophobia are frequently fearful of becoming "ill" 
because of the fear that it might lead to vomiting. The SPOVI also correlated moderately with the OCI suggesting some overlap with the compulsive behaviours (e.g. washing and ruminating) as a means of trying to prevent fear of vomiting. The SPOVI also correlated strongly with symptoms of depression and general anxiety. Depression is probably a consequence of the chronicity and handicap caused by emetophobia over many years and would be expected to be correlated with the SPOVI. The correlation of general anxiety and SPOVI scales may occur from a shared construct such as anxiety sensitivity. In addition people with emetophobia may be in a constant of anticipatory anxiety waiting for their self or others to vomit without any warning.

There were no significant differences between the emetophobia participants recruited in a clinical setting and the Internet, apart from slightly higher health anxiety in the clinical setting. This suggests that there may be factors not measured in the current study that determine treatment seeking such as readiness to change or the confidence in treatment offered. Some of those recruited over the Internet had experienced failed treatments in the past, which is likely to influence any decision on further treatment.

\section{Strengths, Limitations, and Future Directions}

The current study has a number of strengths and new contributions to the scientific literature on emetophobia. The study utilised a sample of 95 individuals with a DSMIV diagnosis of a specific phobia of vomiting. This is among the largest samples of individuals with emetophobia utilised in previous research, and increases our confidence that the findings are likely to be representative of the population of 
individuals with the condition. Comparison with a normative sample allowed for inferences to be made regarding the clinical nature of emetophobic symptoms.

There were limitations in the current research that should be considered when interpreting our results. A significant limitation of the results is the small sample used to assess the sensitivity of the SPOVI to the effect of treatment. Despite its small size, the sample was still sufficient to detect significant differences and give us confidence to use the SPOVI in treatment trials.

The use of a combined clinical group, including individuals diagnosed using a telephone interview, may also reduce certainty in the homogeneity and diagnostic certainty of the clinical group.

Additionally, although our cohorts had a male-female imbalance, this is similar to previous research that has found that the majority of individuals with emetophobia are women (Veale \& Lambrou, 2006; Lipsitz et al, 2001). This imbalance does, however, mean that our results may not be representative of the rarer cases of male emetophobia, few of which were assessed in our method. Despite these limitations, we present these results as an initial investigation that may be replicated in future research. Future studies might administer the scale to a population who regularly experience nausea as a symptom of another anxiety disorder in order to determine whether the scale discriminates between those with Specific Phobia of Vomiting as opposed to those who experience nausea and some fear of vomiting without reaching criterion for a specific phobia. A further limitation is that the scale has not yet been compared to a construct that was theoretically distinct to determine divergent validity.

This study represents the first initial psychometric validation of the SPOVI. Replication of our results in a new sample of individuals with emetophobia would add 
strength to our findings, particularly the latent structure of the symptoms found in our sample. Confirmatory factor analytic procedures would allow for an overall assessment of 'model fit' of our factor structure within a different sample. Such procedures have been used previously to assess the generalisability of a previously derived latent structure in a new sample (e.g., Boschen \& Oei, 2006).

Future research with the SPOVI will need to examine the sensitivity of the measure to treatment effects in a larger sample in a controlled trial, as well as the ability of the measure to distinguish between responders and non-responders. As discussed above, the SPOVI may be used in future research into emetophobia as an assessment of current symptom status and change in these symptoms over time for audit of outcome of emetophobia in national datasets (Clark, Layard, Smithies, Richards, Suckling \& Wright, 2009).

\section{Conclusion}

The current study has validated a brief self-report scale that can be used by clinicians and researchers to assess the symptoms of individuals with a specific phobia of vomiting. There are two subscales of avoidance and threat monitoring. The SPOVI can be used as a tool for treatment planning and outcome measurement and consist of two subscales.

\section{References}

American Psychiatric Association (1994). Diagnostic and statistical manual of mental disorders ( $4^{\text {th }}$ ed.). Washington, DC: Author. 
Antony, M.M. (2001). Measures for specific phobia. In Antony, M.M., Orsillo, S.M., \& Roener, L. (Eds.), Practitioner's guide to empirically based measures of anxiety (pp. 133- 158). New York: Kluwer Academic / Plenum Publishers.

Becker, E.S., Rinck, M., Türke, V., Kause, P., Goodwin, R., Neumer, S., \& Margraf, J. (2007). Epidemiology of specific phobia subtypes: Findings from the Dresden Mental Health Study. European Psychiatry, 22, 69-74.

Boschen, M.J. (2007). Reconceptualizing emetophobia: A cognitive-behavioral formulation and research agenda. Journal of Anxiety Disorders, 21, 407-419.

Boschen, M.J., Veale, D., Ellison, N., \& Reddell, T. (in submission). The Emetophobia Questionnaire (EmetQ-13): Psychometric validation of a measure of specific phobia of vomiting. Manuscript submitted for publication.

Boschen, M.J., \& Oei, T.P.S. (2006). Factor structure of the Mood and Anxiety Symptom Questionnaire does not generalize to an anxious/depressed sample. Australian and New Zealand Journal of Psychiatry, 40, 1017-1026.

Clark, D.M., Layard, R., Smithies, R., Richards, D.A., Suckling, R., \& Wright, B. (2009). Improving access to psychological therapy: Initial evaluation of two UK demonstration sites. Behaviour Research and Therapy, 47, 910-920.

Foa, E.B., Kozak, M.J., Salkovskis, P.M., Coles, M.E., \& Amir, N. (1998). The validation of a new obsessive-compulsive disorder scale: The Obsessive Compulsive Inventory. Psychological Assessment, 10, 206-214.

Haidt, J., McCauley, C., \& Rozin, P. (1994). Individual differences in sensitivity to disgust: A scale sampling seven domains of disgust elicitors. Personality and Individual Differences, 16, 701-713. 
Harvey, A., Watkins, E., Mansell, W., \& Shafran, R. (2004). Cognitive behavioural processes across psychological disorders: A trans-diagnostic approach to research and treatment. Oxford: University Press.

Horn, J. (1965). A rationale and test for the number of factors in factor analysis. Psychometrika, 30, 179-185.

Hunter, P.V., \& Antony, M.M. (2009). Cognitive-behavioral treatment of emetophobia: the role of interoceptive exposure. Cognitive and Behavioral Practice, 16, 84-91.

Jacobsen, N.S., Roberts, L.J., Berns, S.B., \& McGlinchey, J.B. (1999). Methods of defining and determining the clinical significance of treatment effects: Description, application, \& alternatives. Journal of Consulting and Clinical Psychology, 67, 300-307.

Kirkpatrick, D.R, \& Berg, A.J. (1981). Fears of a heterogeneous non-psychiatric sample. Paper presented at the Annual Conference of the American Psychological Association, Los Angeles, California.

Kroenke, K., \& Spitzer, R.L. (2002). The PHQ-9: A new depression diagnostic and severity measure. Psychiatric Annals, 32, 509-515.

Lesage, A., \& Lamontagne, Y. (2003). Paradoxical intention and exposure in vivo in the treatment of psychogenic nausea: Report of two cases. Behavioural Psychotherapy, 13, 69-75.

Lipsitz, J.D., Fyer, A.J., Paterniti, A., \& Klein, D.F. (2001). Emetophobia: Preliminary results of an internet survey. Depression and Anxiety, 14, 149152. 
Lorenzo-Seva, U., \& Ferrando, P. J. (2006). FACTOR: A computer programme to fit the exploratory factor analysis model. Behavioral Research Methods, Instruments and Computers, 38, 88-91.

Manassis, K., \& Kalman, E. (1990). Anorexia resulting from fear of vomiting in four adolescent girls. Canadian Journal of Psychiatry, 35, 548-550.

McFadyen, M., \& Wyness, J. (1983). You don't have to be sick to be a behaviour therapist but it can help! Treatment of a "Vomit" Phobia. Behavioural Psychotherapy, 11, 173-176.

McKenzie, S. (1994). Hypnotherapy for vomiting phobia in a 40 year old woman. Contemporary Hypnosis, 11, 37-40.

Moran, D.J., \& O’Brien, R.M. (2005). Competence imagery: A case study treating emetophobia. Psychological Reports, 96, 635-636.

Mundt, J.C., Marks, I.M., Shear, M.K., \& Greist, J.H. (2002). The Work and Social Adjustment Scale: A simple measure of impairment in functioning. British Journal of Psychiatry, 180, 461-464.

O’Connor, J.J. (1983). Why can’t I get hives: Brief strategic therapy with an obsessional child. Family Process, 22, 201-209.

Olatunji, B.O., Williams, N.L., Tolin, D.F., Abramowitz, J.S., Sawchuk, C.N., Lohr, J.M., et al. (2007). The Disgust Scale: Item analysis, factor structure, and suggestions for refinement. Psychological Assessment, 19, 281-297.

Philips, H.C. (1985). Return of fear in the treatment of a fear of vomiting. Behaviour Research and Therapy, 23, 45-52.

Price, K., Veale, D, Brewin, C.R. (2012). Intrusive imagery in people with a specific phobia of vomiting. Journal of Behavior Therapy and Experimental Psychiatry, 43, 672-678. 
Reddell, T. (2006). Developing the Emetophobia Questionnaire (Unpublished honours thesis). Griffith University, Australia.

Ritow, J.K. (1979). Brief treatment of a vomiting phobia. American Journal of Clinical Hypnosis, 21, 293-296.

Salkovskis, P.M., Rimes, K.A., Warwick, H.M.C., \& Clark, D.M. (2002). The Health Anxiety Inventory: Development and validation of scales for the measurement of health anxiety and hypochondriasis. Psychological Medicine, 32, 843-853.

Spitzer, R.L., Kroenke, K., Williams, J.B.W., \& Löwe, B. (2006). A brief measure for assessing generalized anxiety disorder: The GAD-7. Archives of Internal Medicine, 166, 1092-1097.

van Overveld, M., de Jong, P.J., Peters, M.L., van Hout, W.J.P.J., \& Bouman, T.K. (2008). An internet-based study on the relation between disgust sensitivity and emetophobia. Journal of Anxiety Disorders, 22, 524-531.

Veale, D. (2009). Cognitive behaviour therapy for a specific phobia of vomiting. The Cognitive Behaviour Therapist, 2, 272-288.

Veale, D., \& Lambrou, C. (2006). The psychopathology of vomit phobia. Behavioural and Cognitive Psychotherapy, 34, 139-150.

Veale, D., Costa, A., Murphy, P. \& Ellison, N. (2012). Abnormal eating behaviour in people with a specific phobia of vomiting (emetophobia). European Eating Disorders Review, 20, 414-418

Veale, D, Murphy, P, Ellison, N, Kanakam, N, \& Costa, A (2012). Auto-biographical memories in people with a specific phobia of vomiting. Journal of Behaviour Therapy and Experimental Psychiatry 44, 14-20. 
Wijesinghe, B, (1974) A vomiting phobia overcome by one session of flooding with hypnosis. Journal of Behavior Therapy and Experimental Psychiatry, 5, 169170.

Wilson, P., \& Cooper, C. (2008). Finding the magic number. The Psychologist, 21, 866-867.

Wolitzky-Taylor, K.B., Horowitz, J.D., Powers, M.B., \& Telch, M.J. (2008).

Psychological approaches in the treatment of specific phobias: A metaanalysis. Clinical Psychology Review, 28, 1021-1037.

Zimmerman, M., \& Mattia, J.I. (2001). A self-report scale to help make psychiatric diagnoses: the Psychiatric Diagnostic Screening Questionnaire. Archives of General Psychiatry, 58, 781-794.

Zwick, W.R., \& Velicer, W.F. (1986). Comparison of five rules for determining the number of components to retain. Psychological Bulletin, 99, 432-442. 
Table 1. Demographic Details of the Clinical and Community Samples.

\begin{tabular}{|c|c|c|}
\hline & $\begin{array}{l}\text { Emetophobic } \\
(N=95)\end{array}$ & $\begin{array}{l}\text { Community } \\
(N=90)\end{array}$ \\
\hline Age & $M=32.61, S D=12.09$ & $M=32.47, S D=11.00$ \\
\hline \multicolumn{3}{|l|}{ Sex } \\
\hline Male & $6(6.3 \%)$ & $3(3.3 \%)$ \\
\hline Female & $89(93.7 \%)$ & $87(96.7 \%)$ \\
\hline \multicolumn{3}{|l|}{ Marital Status } \\
\hline Single & $37(38.9 \%)$ & $47(52.2 \%)$ \\
\hline Married / Cohabiting & $53(55.8 \%)$ & $37(41.1 \%)$ \\
\hline Divorced & $4(4.2 \%)$ & $2(2.2 \%)$ \\
\hline Not Recorded & $1(1.1 \%)$ & $4(4.4 \%)$ \\
\hline \multicolumn{3}{|l|}{ Employment Status } \\
\hline Unemployed & $5(5.3 \%)$ & $5(5.6 \%)$ \\
\hline Long-Term Sick Leave & $4(4.2 \%)$ & $1(1.1 \%)$ \\
\hline Student & $15(15.8 \%)$ & $21(23.3 \%)$ \\
\hline Employed & $55(57.9 \%)$ & $55(61.1 \%)$ \\
\hline Homemaker & $7(7.4 \%)$ & $5(5.6 \%)$ \\
\hline Other & $7(7.4 \%)$ & $2(2.2 \%)$ \\
\hline Not Recorded & $2(2.1 \%)$ & $1(1.1 \%)$ \\
\hline
\end{tabular}


Table 2 Factor Loadings and Communality of SPOVI Items.

\begin{tabular}{|c|c|c|c|}
\hline Item & Factor I & Factor II & Communality \\
\hline $\begin{array}{l}\text { I have been avoiding adults or children } \\
\text { because of my fear of vomiting }\end{array}$ & .94 & -.19 & .73 \\
\hline $\begin{array}{l}\text { I have been avoiding objects that other } \\
\text { people have touched because of my fear of } \\
\text { vomiting }\end{array}$ & .83 & -.12 & .59 \\
\hline $\begin{array}{l}\text { I have been avoiding situations or activities } \\
\text { because of my fear of vomiting }\end{array}$ & .81 & -.02 & .64 \\
\hline $\begin{array}{l}\text { I have been looking at others to see if they } \\
\text { may be ill and vomiting }\end{array}$ & .72 & -.01 & .51 \\
\hline $\begin{array}{l}\text { I have escaped from situations because I am } \\
\text { afraid I or others may vomit }\end{array}$ & .58 & .19 & .49 \\
\hline $\begin{array}{l}\text { I have been restricting the amount or type of } \\
\text { foot I eat or alcohol I drink because of my } \\
\text { fear of vomiting }\end{array}$ & .46 & .25 & .40 \\
\hline $\begin{array}{l}\text { I have been trying to avoid or control any } \\
\text { thoughts or images about vomiting }\end{array}$ & .45 & .38 & .53 \\
\hline I have been feeling nauseous & -.27 & 1.00 & .80 \\
\hline $\begin{array}{l}\text { If I think I am going to vomit, I do } \\
\text { something to try to stop myself from } \\
\text { vomiting }\end{array}$ & -.16 & .87 & .64 \\
\hline $\begin{array}{l}\text { I have been trying to find reasons to explain } \\
\text { why I feel nauseous }\end{array}$ & -.04 & .80 & .62 \\
\hline
\end{tabular}




\begin{tabular}{|c|c|c|c|}
\hline $\begin{array}{l}\text { I have been focussed on whether I feel ill } \\
\text { and may vomit, rather than on my } \\
\text { surroundings }\end{array}$ & .12 & .68 & .57 \\
\hline $\begin{array}{l}\text { I have been worrying about myself or others } \\
\text { vomiting }\end{array}$ & .23 & .60 & .56 \\
\hline $\begin{array}{l}\text { I have been thinking about how to stop } \\
\text { myself or others from vomiting }\end{array}$ & .31 & .55 & .57 \\
\hline $\begin{array}{l}\text { I have been seeking reassurance that I or } \\
\text { others will not be ill and vomit }\end{array}$ & .32 & .53 & .57 \\
\hline
\end{tabular}

Note. Loadings > .40 are displayed in bold 
Table 3.

Age and Scores for the Clinical Cohorts and Community Controls

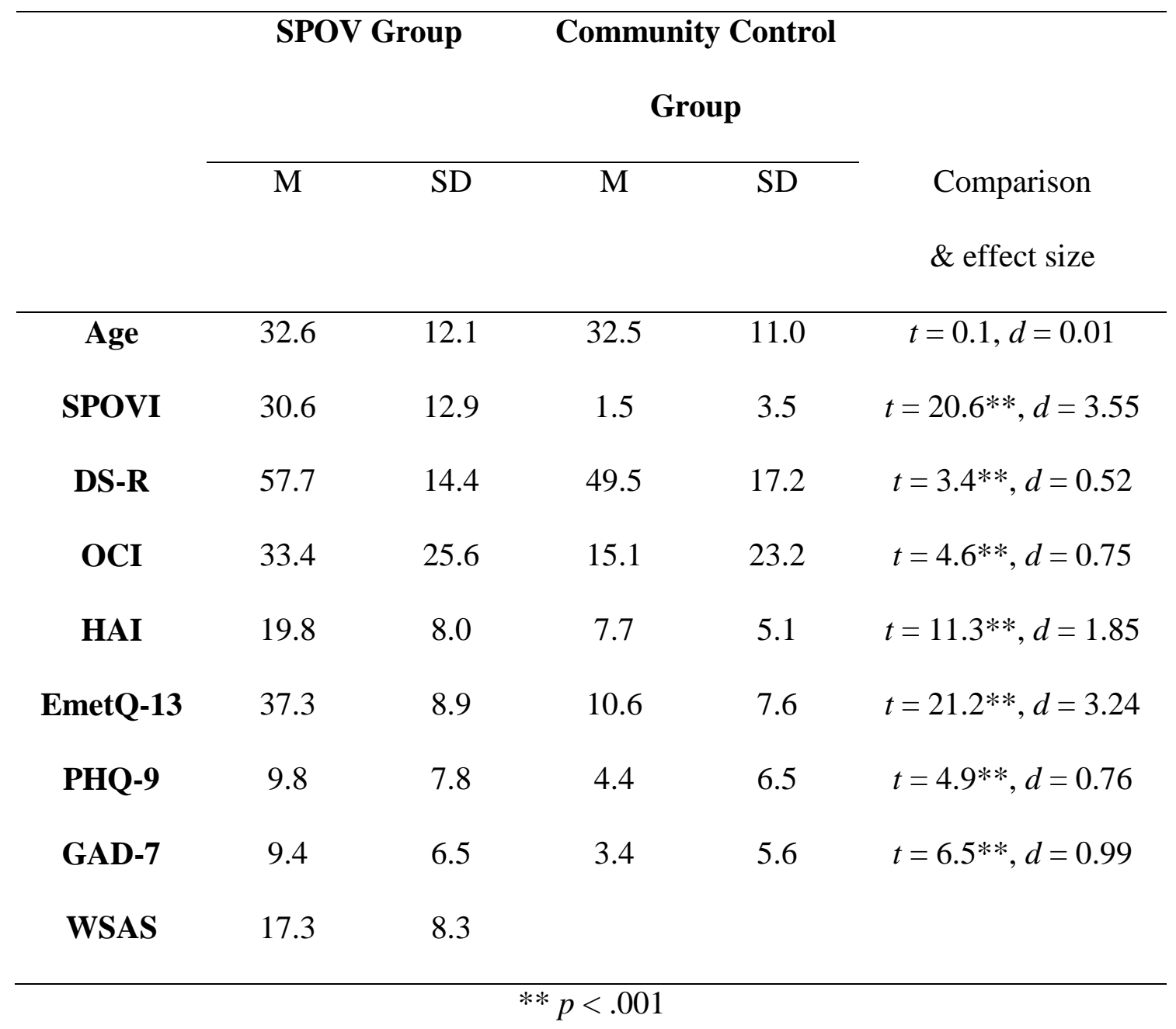


Table 4.

Sensitivity and Specificity of SPOVI Cutoff Scores

\begin{tabular}{ccc}
\hline SPOVI Cutoff Score & Sensitivity & Specificity \\
\hline$>\mathbf{5}$ & 1.00 & .92 \\
$>\mathbf{1 0}$ & .97 & .96 \\
$>\mathbf{1 5}$ & .84 & .98 \\
$\mathbf{> 2 0}$ & .75 & 1.00 \\
$>\mathbf{2 5}$ & .65 & 1.00 \\
\hline
\end{tabular}

